\title{
Fuzzy Expression of Hand-drawn Paths in We-Map Environment
}

\author{
SHEN Ruru ${ }^{a, b}$, YAN Haowen ${ }^{\text {a,b,*, LU Xiaomin }}{ }^{\text {a,b }}$, SUN Qinke ${ }^{a, b}$ \\ ${ }^{a}$ Faculty of Geomatics, Lanzhou Jiaotong University, Lanzhou 730070, China, 1050182127@qq.com, H.Yan, \\ haowen2010@gmail.com,X.Lu,xiaominlu08@gmail.com,Q.Sun,874694642@qq.com \\ ${ }^{b}$ Gansu Provincial Engineering Laboratory for National Geographic State Monitoring, Lanzhou730070, China, \\ 1050182127@qq.com,H.Yan, haowen2010@gmail.com ,X.Lu,xiaominlu08@gmail.com,Q.Sun,874694642@qq.com \\ * Corresponding author
}

\section{Keywords: We-Map; Hand-drawn paths; Fuzziness}

Abstract: We-Map is a "grassroots" map for the general public and the hand-drawn paths function makes it a kind of "Personal Media" with strong interactivity. The hand-drawn paths are manually drawn by the "grassroots" public and intuitively show the paths to the destination. The fuzzy expression of the hand-drawn paths has low requirements on direction, distance and topological relationship. Compared with traditional navigation with high precision requirements, its expression is more easily accepted by the "grassroots".

In real life, the public's perception of geographic navigation is biased towards fuzziness and templating. Taking the location of the China National Stadium (Bird's Nest) as an example, the "grassroots" public is usually expressed near the Beijing Olympic Park or by subway line 15 or subway line 8 at the exit of the Olympic Park Station D. This expression is different from the traditional Accurate and standardized navigation. Therefore, this paper combines a new generation of We-Map concept with map as the information carrier, expresses the recommended path from the departure point to the destination in the form of hand-drawn maps fuzzification and template, and explores and designs a model suitable for mobile devices.

The basic ideas are as follows: (1) Determining the place of departure and destination. (2) The expression design of the hand-drawn path is fuzzy: firstly, the projection and calculation of the fitting path, and then extracting the multi-level feature points of the facilities around the road by using the feature points extraction principle. Finally, corner calculations and template nesting at the corners. (3) The definition of fuzzification is as follows: "starting from the starting point, follow the XX road, go straight to the XX place near XX, turn right/left to XX road, and to the XX place near XX, reach the end point". The template can be expressed as: " starting point, along the line, turn, end point ". (4) Matching multiuser and multi-map versions to hand-drawn maps with fuzzified expression. Take a case from this model: departure at the entrance of Feitian Hotel, Lanzhou City, Gansu Province, and destination at Cold and Arid Regions Environmental and Engineering Research Institute, Chinese Academy of Sciences.

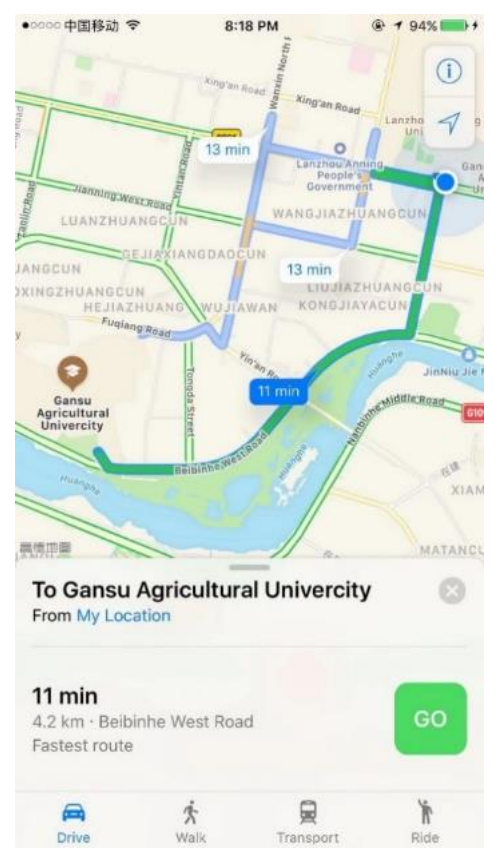

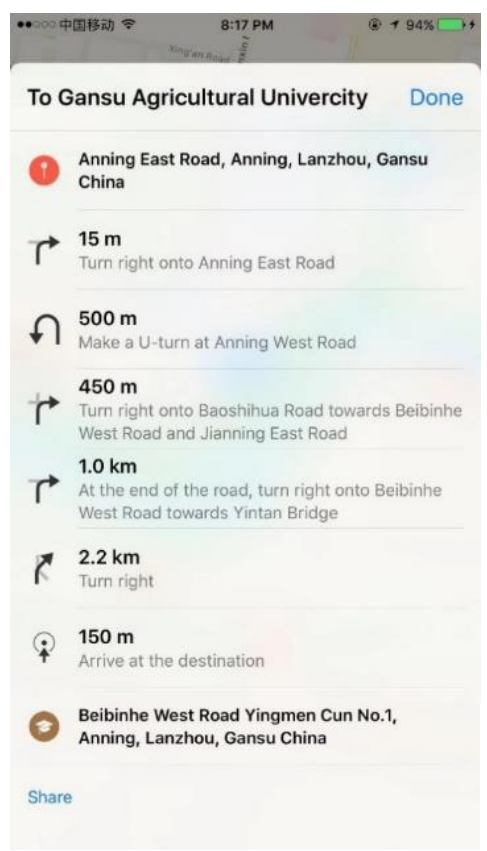

Note: Corresponding disadvantages of traditional navigation maps: the "grassroots" public can't understand the southeast and northwest, or can't feel $450 \mathrm{~m} \cdots$

Figure1. Gaode navigation map from Lanzhou Jiaotong University

to Gansu Agricultural University 


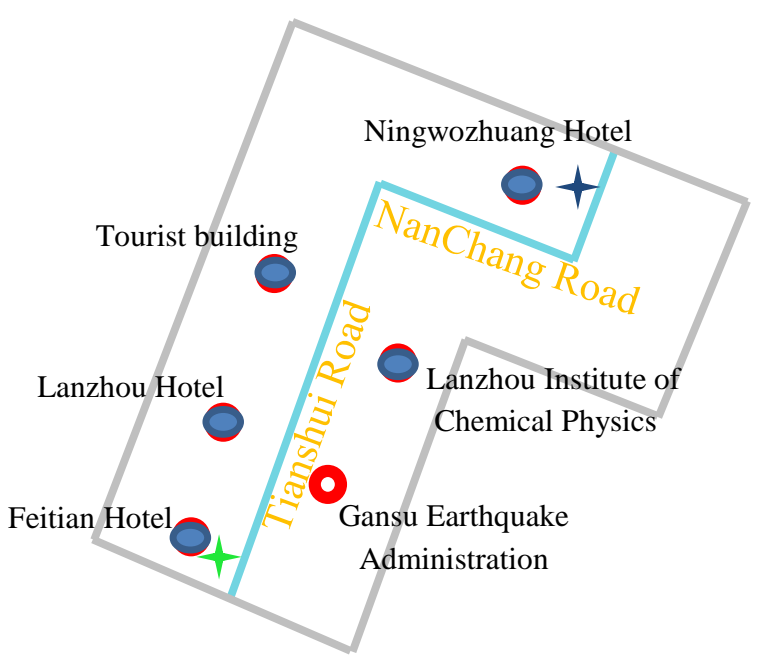

Figure a. First level (Near XX)

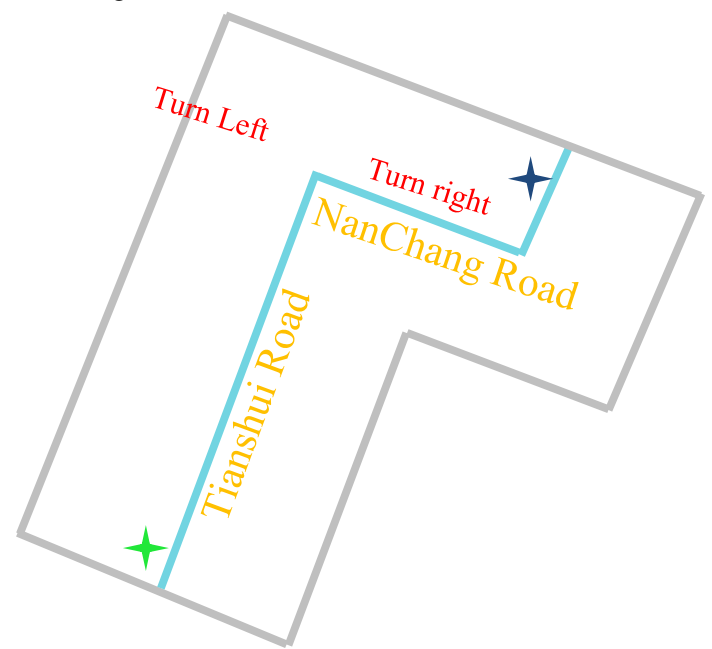

Figure c. Corner calculation

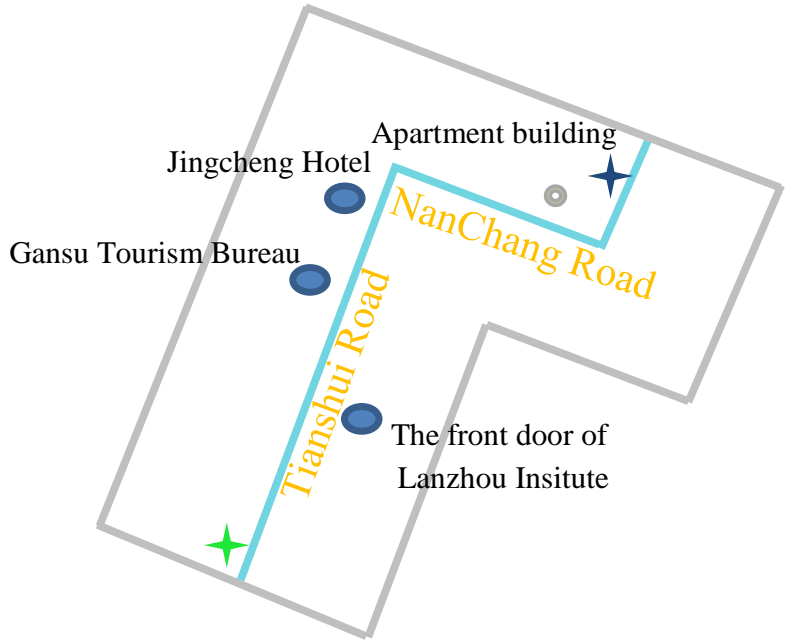

Figure b. Second level (XX position)

Note: $(1)+$ is the starting point

(2) $\downarrow$ is the destination point

(3) Figure a and Figure b are user-selected step-by-step feature points

(4) $=$ is the road

- is the buffer of the line road

\section{To Cold and Arid Regions Environmental and Engineering Research Institute Done}

\section{¿ Feitian Hotel, Lanzhou City, Gansu}

$\rightarrow$ Go straight along the Tianshui Road to the nearby Lanzhou Hotel

$\rightarrow$ Go straight through the nearby Lanzhou Institute of

$\rightarrow$ Chemical Physics

$\rightarrow$ Go straight through the Gansu Tourism Bureau near the Tourist Building

$\rightarrow$ At Jincheng Hotel, turn right at Nanchang Road

$\rightarrow$ Go straight along the Nanchang Road to the nearby Ningwozhuang Hotel

$\rightarrow$ Turn left at the location of apartment building of Lanzhou Institute of Chemical Physics

Destination

Example 